migrant population from the Indian Subcontinent. A more detailed analysis of migration patterns and its impact on UK cases of TB is warranted.

\section{S40 A CROSS SECTIONAL INVESTIGATION TO DETERMINE THE BACKGROUND PREVALENCE OF LATENT TUBERCULOSIS INFECTION IN UNSELECTED MEDICAL INPATIENTS IN A LOW PREVALENCE REGION OF UK REVEALS HIGH RATES OF IGRA POSITIVITY}

doi:10.1136/thoraxjnl-2011-201054b.40

${ }^{1} \mathrm{~N}$ Varsani, ${ }^{2} \mathrm{~T}$ S C Hinks, ${ }^{2} \mathrm{D}$ T Godsiff, ${ }^{2} \mathrm{~T}$ C Bull, ${ }^{3} \mathrm{~K}$ L Nash, ${ }^{3} \mathrm{~L}$ McLuckie, ${ }^{3} \mathrm{~A}$ Warley. ${ }^{1}$ St George's University of London, London, UK; ${ }^{2}$ Department of Infection, Inflammation and Immunity, University of Southampton School of Medicine, Southampton, UK; ${ }^{3}$ Salisbury NHS Foundation Trust, Salisbury, UK

Introduction The background rate of latent tuberculosis infection (LTBI) in low prevalence regions of the UK is unknown. Interferon $\gamma$ release assays (IGRAs) are sensitive and specific methods for detecting LTBI, and have accurately characterised the epidemiology of LTBI among high risk populations such as recent TB contacts or immigrants. However there are no current data on the incidence of IGRA positivity among the general adult population in the UK. Such data would be valuable for interpreting the significance of a positive IGRA result, and guiding cost-benefit analyses of new diagnostics. Methods A TB outbreak occurred within a rural DGH. 481 individuals were identified as potential contacts and were tested by IGRA

Abstract S40 Table 1

\begin{tabular}{|c|c|c|c|c|}
\hline & \multicolumn{4}{|c|}{ Unexposed, age-matched control patients } \\
\hline & $\begin{array}{l}\text { TSpot positive } \\
n=13 \\
n(\%)\end{array}$ & $\begin{array}{l}\text { TSpot negative } \\
\mathrm{n}=135 \\
\mathrm{n}(\%)\end{array}$ & OR & Univariate $\mathrm{p}$ \\
\hline Age (years), median (range) & $64(45-87)$ & $70(25-93)$ & & 0.81 \\
\hline Male, n (\%) & $5(38)$ & $70(52)$ & & 0.36 \\
\hline \multicolumn{5}{|l|}{ Ethnicity, n (\%) } \\
\hline White Caucasian & $13(100)$ & $135(100)$ & & \\
\hline \multicolumn{5}{|l|}{ Country of birth } \\
\hline UK born & $9(69)$ & $125(93)$ & 0.18 & 0.006 \\
\hline Other & $4(31)^{*}$ & $9(6.7)$ & & \\
\hline $\begin{array}{l}\text { Years since immigration, } \\
\text { median (range) }\end{array}$ & $43(21-62)$ & $52(8-61)$ & & \\
\hline $\begin{array}{l}\text { Ever visited a high prevalence } \\
\text { country, n (\%) }\end{array}$ & $4(31)$ & $46(37)$ & & 0.84 \\
\hline $\begin{array}{l}\text { Years since last visit, } \\
\text { median (range) }\end{array}$ & $8.5(0-40)$ & $5.5(0-60)$ & & \\
\hline $\begin{array}{l}\text { Ever resident in a high prevalence } \\
\text { area }>6 / 52, n(\%)\end{array}$ & $2(15)$ & $27(20)$ & & 0.64 \\
\hline $\begin{array}{l}\text { Occupation: healthcare, prison, } \\
\text { lab n }(\%) \dagger\end{array}$ & $4(31)$ & $18(13)$ & & 0.09 \\
\hline \multicolumn{5}{|l|}{ BCG (history or scar), $\mathrm{n}(\%)$} \\
\hline Yes & $7(54)$ & $66(49)$ & & 0.73 \\
\hline No & $4(31)$ & $44(33)$ & & \\
\hline Unknown & $2(15)$ & $25(19)$ & & \\
\hline \multicolumn{5}{|l|}{ Medical history, n (\%) } \\
\hline $\begin{array}{l}\text { Comorbidity known to be } \\
\text { associated with TB } \neq\end{array}$ & $0(0)$ & $21(16)$ & & 0.22 \\
\hline Immunosuppresive medications§ & $4(31)$ & $12(9.2)$ & 4.6 & 0.015 \\
\hline Ever known to be exposed to TB & $4(31)$ & $26(19)$ & & 0.32 \\
\hline Ever had treatment for tuberculosis & $2(15)$ & $2(1.5)$ & 12 & 0.003 \\
\hline
\end{tabular}

*Germany (2), South Africa (1), Hong Kong (1).

†Percentages are those of those with valid data (eg occupation).

$\ddagger$ Diabetes mellitus (17), chronic renal failure (3), haematological malignancy (2), gastric surgery (1).

§Systemic steroids (12), methotrexate (4), hydroxychloroquine (2), anti-TNF- $\alpha$ (2), sulfasalzine (1).
(TSpot.TB). Uniquely, for comparison, we recruited an additional large cohort of age matched controls from the same general wards but with no exposure to the outbreak.

Results 456 staff and patients were tested including 148 unexposed age-matched patient controls. Rates of positivity were $22 \%(95 \% \mathrm{CI}$, 14 to 29 ), $11 \%$ (6.1 to 16 ), $8.8 \%$ (4.2 to 13 ) and $9.5 \%$ (3.0 to 22 ) among exposed patients, exposed staff, unexposed patients and unexposed staff respectively. 8 cases of active TB (identical VNTR profile) and an estimated 35 cases of recently acquired LTBI can be attributed to exposure to the index case, out of 481 contacts. Characteristics of the unexposed controls are in Abstract S40 table 1. IGRA positivity was associated in multivariate analyses with history of previous TB treatment ( $O R$ 11, $p=0.04$ ) and use of corticosteroids (OR 5.9, $\mathrm{p}=0.02$ ), but not with age. The age specific prevalences of IGRA positivity were 0 (N/A) for ages $<40,15.3 \%$ (12.2 to 29.4 ) for ages $40-59,7.0 \%$ (0.92 to $13 \%$ ) for age $60-79$, and $10 \%(5.9$ to 19$)$ for ages $=80$.

Conclusions We observed a surprisingly high background rate of IGRA positivity among an unselected population typical of respiratory and general medical inpatients in a rural DGH. All controls were white-Caucasians, who comprise $92 \%$ of the UK population, and may represent a current minimum UK background rate. As rates were highest in the 5th and 6th decade, in the context of ageing populations and increasing iatrogenic immunosuppression, reactivation of LTBI may be a persistent hazard for several decades to come.

\section{S41 SCREENING FOR LATENT TB IN HIV: ARE NICE \& BHIVA GUIDANCE EFFECTIVE?}

doi:10.1136/thoraxjnl-2011-201054b.41

S Capocci, C Smith, I Cropley, S Bhagani, M A Johnson, M C I Lipman. Royal Free Hospital, London, UK

HIV infection is the strongest single risk factor for the development of active TB in latently infected individuals. NICE and BHIVA 2011 guidelines both recommend screening for latent TB in HIV positive subjects in the UK. NICE bases this on blood CD4 count; and recommends testing all patients with $\mathrm{CD} 4<500$ cells/ $\mu \mathrm{l}$ (if CD4 $<200$, this is with an Interferon Gamma Release Assay (IGRA) and tuberculin skin test (TST), and if CD4 200-500, then IGRA \pm TST is proposed). BHIVA test-stratify on CD4 count, country of origin and use of antiretroviral therapy (ART). They recommend IGRA in the following populations: all subjects from Sub Saharan Africa, irrespective of CD4 if on ART $<2$ years; medium TB incidence countries if on ART $<2$ years and CD4 $<500$; and low TB incidence countries if on ART $<6$ months and CD $4<350$. To our knowledge, neither strategy has been formally tested in a UK HIV population. Here, using data on all subjects over a 10-year period (2000-2010) within our large HIV service ( $n=3306)$, we determine the impact of applying both strategies to detect cases of latent TB infection who subsequently developed active TB during this time period $(n=72)$. Subjects who developed active $T B<3$ months from HIV diagnosis were excluded from analysis, as it was felt that the

\section{Abstract S41 Table 1}

\begin{tabular}{lll}
\hline & NICE & BHIVA \\
\hline Eligible for screening & 2778 & 1478 \\
Number eligible, who developed TB (PPV) & $66(2.4 \%)$ & $42(2.8 \%)$ \\
Not eligible for screening & 528 & 1828 \\
Number not eligible, who developed TB & $6(1.1 \%)$ & $30(1.6 \%)$ \\
NPV & $98.9 \%$ & $98.4 \%$ \\
Sensitivity & $92 \%$ & $58 \%$ \\
Specificity & $16 \%$ & $56 \%$ \\
\hline
\end{tabular}

Note: PPV $=$ positive predictive value, NPV = negative predictive value 
time interval was too short for them to be screened for TB. Abstract S41 table 1 illustrates the differences between NICE and BHIVA strategies over the 10-year period. Neither screening method had optimal sensitivity and specificity. Both guidelines' eligible groups were only twice as likely to develop active TB compared to those ineligible for screening. NICE guidelines were poorly specific while the BHIVA strategy missed $42 \%$ of cases (largely as they were either UK born or on ART). Using data from our UK HIV population, we find that new TB screening guidance does not appear to discriminate those at risk of active $\mathrm{TB}$.

\section{S42 EARLY IDENTIFICATION OF MYCOBACTERIUM TUBERCULOSIS (M.tb) AND MYCOBACTERIUM OTHER THAN TUBERCULOSIS (MOTT) BY "CORD FORMATION" EVALUATION IN LIQUID CULTURE}

doi:10.1136/thoraxjnl-2011-201054b.42

A Elsaghir, S Abdullah, F Sundram, D D Creer. Barnet and Chase Farm Hospitals NHS Trust, London, UK

The identification of M.tb in smear negative samples has the inherent TB culture delays. These delays potentially affect treatment choices and contact tracing decisions particularly where TB prevalence is low and isolation of MOTT common. Faster molecular techniques are expensive and not universally available. The identification of cord formation ${ }^{1}$ in positive cultures gives an early indication of the likely $\mathrm{TB}$ culture result before the final reference laboratory identification (local audit data: M.tb 12.3 \pm 5.9 d, MOTT $13.7 \pm 11.6 \mathrm{~d}$ ). We evaluated the presence of cord formation in AFB smears prepared from MGIT tubes and stained by auramine-phenol staining from positive liquid culture (BACTEC MGIT growth supplement medium) for the presumptive identification of $M . t b$. We prospectively evaluated 612 positive mycobacterial culture specimens from 316 patients over 3 years. All specimens were sent to our local reference laboratory for species identification. The identification of $M . t b$ by the presence of cord formation was compared with the subsequent reference laboratory report. 420/426 samples showing cording by smear microscopy were confirmed by the reference laboratory as M.tb (PPV: 98.6\%). 182/186 non-cording culture positive specimens were confirmed as MOTT (NPV: 97.9\%). The $6 / 612(0.9 \%)$ MOTT misidentified as $M . t b$ by cording were $M$ kansasii as previously described. ${ }^{1}$ The $4 / 612(0.7 \%)$ M.tb misidentified as MOTT by the absence of cording were all $3+$ sputum smear positive samples as previously described. ${ }^{2}$ The presumptive identification of $M . t b$ and MOTT by the presence or absence of cording formation in liquid culture is both sensitive and specific. This technique has a potentially significant impact on treatment timing and contact tracing decisions.

\section{REFERENCES}

1. McCarter YS, et al. Cord formation in BACTEC medium is a reliable, rapid method for presumptive identification of Mycobacterium tuberculosis complex. J Clin Microbiol 1998;36:2769-71.

2. Koksalan $\mathbf{O K}$, et al. Reliability of cord formation in BACTEC 12B/13A media for presumptive identification of Mycobacterium tuberculosis complex in laboratories with a high prevalence of Mycobacterium tuberculosis. Eur J Clin Microbiol Infect Dis 2002;21:314-17.

\section{S43 POLYFUNCTIONAL T CELLS REVEAL THE SPECTRUM OF TUBERCULOSIS IN HIV CO-INFECTION THROUGH THE IDENTIFICATION OF IMMUNOLOGICAL CORRELATES OF LATENT AND ACTIVE DISEASE}

doi:10.1136/thoraxjnl-2011-201054b.43

${ }^{1} \mathrm{~K}$ M Pollock, ${ }^{1} \mathrm{D}$ Montamat-Sicotte, ${ }^{2} \mathrm{G}$ Cooke, ${ }^{1} \mathrm{M}$ Kapembwa, ${ }^{3} \mathrm{O} \mathrm{M}$ Kon, ${ }^{2} \mathrm{G}$ P Taylor, ${ }^{1} \mathrm{~A}$ Lalvani. ${ }^{1}$ Department of Respiratory Medicine, Tuberculosis Research Unit, National
Heart and Lung Institute, Imperial College London, London, UK; ${ }^{2}$ Department of Medicine, Division of Infectious Diseases, Imperial College London, London, UK ${ }^{3}$ Tuberculosis Service, St Mary's Hospital, Imperial College Healthcare Trust, London, UK

Background Approximately one-third of the world's population is infected with Mycobacterium tuberculosis (MTB), mostly as latent tuberculosis infection (LTBI). HIV co-infection confers the single greatest risk of progression to active tuberculosis (TB) and thus provides an opportunity to study the host-pathogen interaction in LTBI. The stage of TB disease and effect of advancing HIV infection should be reflected in the phenotype and function of MTB-specific T cells.

Aims To compare MTB-specific CD4+ and CD8+ Tcell responses in donors from four clinical phenotypes (TB/HIV, TB, LTBI/HIV and LTBI) and test whether TB in the context of HIV is a spectrum of disease dependent on mycobacterial load consequent upon advancing immunosuppression and viraemia.

Methods Donors $(n=34)$ with or without HIV co-infection were carefully selected for active TB or LTBI. Peripheral blood mononuclear cells (PBMCs) were stimulated overnight with purified protein derivative (PPD) or a combination of MTB-specific antigens. An 11-colour intracellular cytokine secretion assay was used to assess CD3, CD4, CD8, IFN- $\gamma$, IL-2 and TNF- $\alpha$ expression.

Results The proportion of participants with a CD8+ PPD-specific IFN- $\gamma(p=0.001)$ or TNF- $\alpha(p=0.006)$ response closely mirrored stage of TB disease, whereas the equivalent CD4 response was unaffected. The proportion of $\mathrm{CD} 4+$ and $\mathrm{CD} 8+\mathrm{PPD}$-specific cells increased with TB stage for IFN- $\gamma$ and TNF- $\alpha$, but did not increase in HIV/TB co-infection compared with TB (Abstract S43 figure 1). When individual cytokine subsets were examined, the percentage of
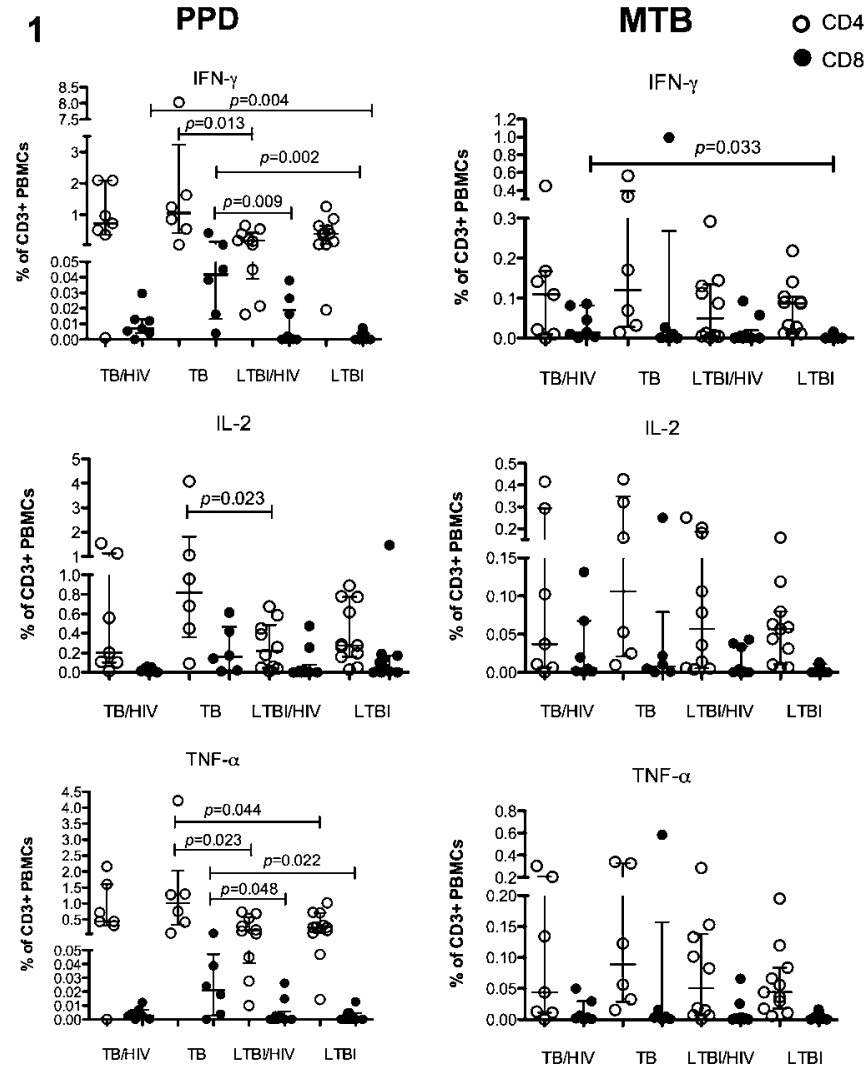

Abstract S43 Figure 1 The proportion of CD3 + CD4+ and CD8+ cells with a total cytokine response to PPD or MTB antigens following $16 \mathrm{~h}$ of stimulation. PBMCs were stained with an 11-colour panel including a dead cell marker and fluorochrome-conjugated antibodies for CD3, CD4, CD8, IFN- $\gamma$, IL-2 and TNF- $\alpha$ and acquired on an LSR-II flow cytometer. 